\title{
Application of Innovative Design Thinking in Product Design* Intelligent Waste Paper Recycling Machine Design Case
}

\author{
Jun $\operatorname{Han}^{\dagger 1, a}$, LuYao Gu${ }^{\dagger 1, b}$, DeRun Chen ${ }^{1, c}$ \\ ${ }^{1}$ School of Art \& Design Wuhan Institute of Technology Hubei China
}

\begin{abstract}
With the rapid development of society and economy, the level of technology and culture in our country is constantly improving, and the content of product design extends from the product appearance design to the design of a whole system of product function, structure, material, production crafts, marketing, maintenance and recycling. The product innovation design thinking should not only consider a single factor, but also integrate the latest achievements in all aspects of the product design ecosystem. The research on innovative thinking and innovative design methods from all walks of life is also constantly systematized and theorized, but the innovative design thinking of products has different thinking characteristics and methods from other fields. This article combines product design examples of intelligent waste paper recycling machines to explore the application of innovative thinking methods in product design.
\end{abstract}

\section{Introduction}

Innovative design thinking is a purposeful thinking process. The essence of innovative design is to meet people's actual needs and potential needs. ${ }^{1}$ As designers, they should continue to carry out innovative designs to meet people's reasonable needs for society, life, and development, and use innovative products to achieve design goals. Innovative design thinking will continue to develop in accordance with changes in society, market environment, scientific and technological level, and customer demand trends. How to use the existing technological and cultural achievements to integrate the best product solutions that meet the needs of society requires a set of systematic and feasible product innovation design thinking and methods to support the design activities. In a broad sense, innovative design thinking can be new discoveries, new ideas, new plans, or new forms of expression, new expression methods, the thinking and formation process of new product schemes. The research on product innovation design thinking in this article mainly focuses on sorting out, summarizing and applying the entire design process of product innovation.

\section{Design thinking criteria for product innovation}

One of the world's leading design schools-Stanford D.School promotes the "Design Thinking" design principle, which divides design thinking into 5 major steps: "Empathy ", "Define", "Iideate", "Prototype" and "Test".2 As a way of thinking that can act on different objects and solve various design problems, design thinking does not come out of thin air, but evolved from traditional design methodology. This design thinking makes the innovative design process of products no longer whimsical and aimless, but becomes a set of general, logical, and systematic problem-solving processes. The following is a brief description of the five steps of "Design Thinking" (Figure 1).

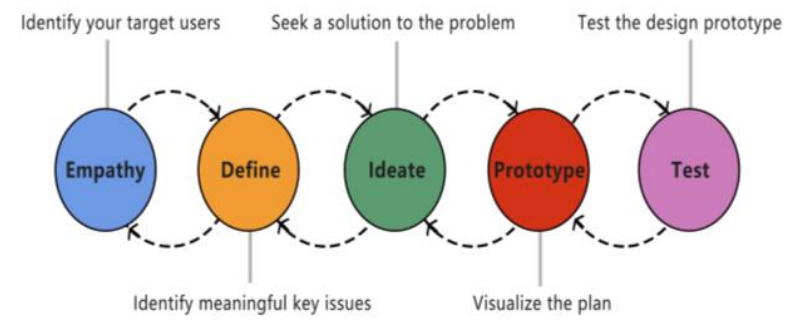

Figure 1: Five steps of design thinking

\subsection{Empathize}

Empathize is usually translated as "identifying with others" in product design, that is the ability to empathize with other people's feelings or emotions. It emphasizes "people-centered" in design thinking, understanding the needs of target users is its core goal and also the core link in the whole design thinking criteria. The purpose of this step which mainly uses the methods of observation, listening and integration is to understand the user's real ideas as much as possible and lay the foundation for the following definition and conception. 


\subsection{Define}

After understanding user's requirements in the previous step, the design team should find out the demand points from these information, and analyze, process and summarize them in the form of "point of view (POV)". Finally, the design team identifies key issues that are meaningful and feasible.

\subsection{Ideate}

The members of design team can use various innovative design methods such as brainstorming, attribute enumeration, reverse analysis, etc. considering the people, objects and environment that the product design project may involve and seeking as many solutions as possible. Finally, the design team determines an optimal product concept scheme after a series of concept creation processes such as analysis, comparison, and screening.

\subsection{Prototype}

The Prototype stage further visualizes the ideas obtained above, it can get closer and closer to the needs of users and gradually generate better solutions through continuous creation, testing, revision and iteration of models. In addition to making product prototypes, Prototype also emphasizes that people discover and explore new problems and solutions in the process of making prototypes.

\subsection{Test}

The target population will test the design prototype in this step. The design team obtains user feedback by obtaining user participation experience, asking open questions, observation, etc. On this basis, they re-examine, analyze, and improve the conceptual scheme to continuously improve the product solutions.

\section{Brief introduction of innovation design method}

Innovative design method is a summary of the law of innovation in design practice and a concrete practical means of innovative thinking. ${ }^{3}$ Designers can find innovation opportunities more quickly and accurately by using specific innovative design methods in the product design process, So as to further create high-quality products that meet user needs. The following is a brief introduction to several innovative product design methods widely used in modern society.

\subsection{Brainstorming}

Brainstorming, also known as group wisdom concentration method, is a method of exerting collective wisdom. The specific operation process of this method is to first inform the members of the design team of the product innovation requirements and conditions, and each member can boldly propose personal ideas after thinking. This method can obtain a large number of conceptual schemes in a short period of time, but most of the schemes will be eliminated due to lack of feasibility or creativity in the later discussion process. This method is widely used in various design practices and is a relatively mature innovative method.

\subsection{Reverse thinking method}

The essence of the reverse thinking method is to "think the other way around", which is a way of thinking that breaks away from inertia and breaks the routine. It does not use traditional viewpoints to define design objects, but uses new perspectives and ways of thinking to analyze problems and propose solutions to problems. The idea of GPS is based on the reverse thinking of the relationship between satellite and ground signal tracking. This bold idea has produced positioning technology that is widely used in our lives.

\subsection{Displacement technology innovation method}

This method refers to the transfer of advanced technology in one field to another field or the application of advanced technology in one product to another product to obtain a new product. ${ }^{4}$ Many large companies in our country have used this method and achieved great success.

\subsection{Attribute enumeration method}

The attribute enumeration method was proposed by Professor Crawford of the University of Nebraska in 1954. This method emphasizes listing and analyzing the attributes of object verbs, nouns, adjectives and so on in the process of innovation, and proposes suggestions for improvement or changes to each attribute. In this way, designers can understand design objects more systematically and clearly, and realize further innovation.

\subsection{Extreme user research method}

The extreme user research method emphasizes focusing on extreme users, that is focusing on certain people with weak or strong abilities. Through in-depth research, we can make design improvements and innovations based on discovering the needs of these users. For example, the various physical functions of the elderly gradually weaken with age. Some modern smart phones have also introduced the elderly phone mode to cater to the physical condition and behavior habits of the elderly, and added functions such as font enlargement and voice broadcast.

\section{Case analysis of intelligent waste paper recycling machine}

In the initial stage of the design project, our team's goal is to design a product that "can effectively solve the problem of the proliferation of urban waste paper flyers". In order to better combine theory and practice, this article analyzes 
the entire design process of the smart waste paper recycling machine based on the "design thinking" method. The application of innovative design thinking in the entire product design process that verifies the significance and value of this thinking method in product design.

\subsection{The "empathetic thinking" stage}

First of all, empathetic thinking is carried out on the design objects, and the design team mainly used methods such as observation, listening, and integration to understand the real needs of the target users. Aiming at the design goal of "can effectively solve the problem of the proliferation of urban waste paper flyers", the team began to observe the phenomenon of randomly discarding waste paper flyers. Through field investigations, it was found that shopping malls, communities, and schools were the most serious places of this phenomenon. It caused a large amount of urban garbage and seriously affected the appearance of the local city.

The design team continued to focus on the people related to the design objects and selected several typical shopping malls, schools, and communities in the local area, and conducted observations, interviews and other interactions with local people, trying to understand the real thoughts and personal needs of users. During the site visit, the design team found many problems caused by waste paper flyers. Most people who distribute flyers think that the distribution of flyers is difficult and the publicity efficiency is low, but the distribution of flyers does not require advanced knowledge and skills, and they are helpless with "self-made garbage". Merchants said that flyers have the advantages of strong pertinence, wide coverage, obvious informing effect, and relatively low cost compared to other forms of publicity, and it is unlikely that they will give up this form of publicity. Many customers say that these overwhelming paper advertisements have affected their normal lives and make them annoying, and some illegal advertisements may even harm the rights of consumers. Local citizens said that these paper flyers not only waste paper resources, affect the appearance of the city, but also cause environmental pollution. The local cleaning staff believed that the paper flyers were difficult to clean and sort, and the flyers on the ground also increased their workload. Especially on a rainy day, the paper flyers were washed by rain and shattered into small pieces which fit tightly on the ground, bringing cleaning staff a lot of trouble. They are eager to have a special flyers recycling equipment to help them reduce the workload.

In this survey, $46 \%$ of people said that they are willing to do such environmentally friendly recycling behavior subjectively without effective incentives, but may not act according to their own subjective wishes. $43 \%$ of people said existing recycling equipment is cumbersome to operate and lack interesting, and the experience is poor. $82 \%$ $\mathrm{t}$ of them said they would be happy to collect and recycle their waste paper if it were convenient and fun.

\subsection{The "Requirements Definition" stage}

Through the analysis of the above research information and data, team members found out the two demand points of "giving corresponding incentive measures" and "making the recycling process more interesting" from these information. The design team further analyzes the demand point to determine the final key problems to be solved, the design ideas are presented in the form of charts which can provide references for the subsequent design process (Figure 2).

The target objects determined by the team are people of all ages, mainly focusing on children and teenager, and the places where the products are used are mainly shopping malls, schools, communities and other places with high traffic. The key issues are summarized as follows: First, design a waste paper recycling machine that can realize efficient recycling and reuse of waste paper; Second, increase the fun and participation of recycling equipment; Third, let waste paper The recycling process becomes the experience process of "reuse of waste paper"; Fourth, give certain incentive feedback to increase the public's enthusiasm for waste paper recycling.

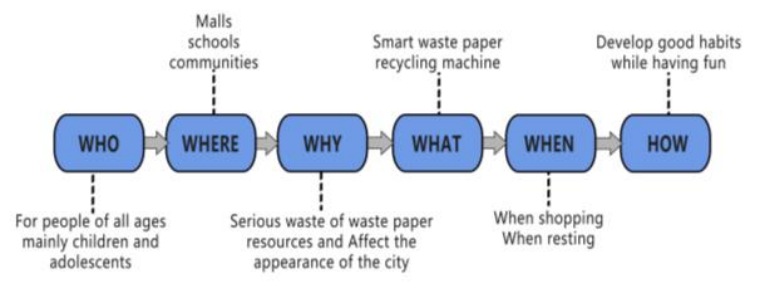

Figure 2: Design Ideas of Waste Paper Recycling Equipment

\subsection{The "Creative Ideas" Stage}

In the stage of " creative Ideas", the design team conceived the conceptual scheme through the above mentioned brainstorming, Displacement technology innovation method, extreme user research and other product innovation design methods. Early in the creative conception, team members already have a deep understanding of the user and the problem to be solved. Therefore, the team can come up with many solutions through brainstorming, some of them seem impossible to implement, but we also put them on the table for discussion. Finally, we came up with two excellent solutions that each team member agreed with emotionally and intellectually, based on the comparison, selection, classification, and combination of many product solutions.

The design team got two solutions after the above series of processes: first, through the combination of interactive stereoscopic wall and waste paper recycling machine, each process of "traditional paper making" is displayed when users are recycling waste paper, which gives users the experience of recycling waste paper, and users can get self-made postcards or business cards after experiencing the process (Figure 3); Secondly, virtual reality technology is applied to waste paper recycling machines to simulate the real "paper making process" through the three-dimensional dynamic interaction between humans and machines. The user will eventually 
get a commemorative self-made postcard, and the recycled paper will also be compressed, stored and finally transported to a fixed paper mill by a dedicated person for recycling (Figure 4). After a joint discussion among the team members, everyone believed that handing over the waste paper recycling process to a professional paper mill would further reduce development costs. In the process of implementing the first scheme, the following issues must also be considered, such as the water and electricity of the recycling equipment, the final waste water discharge and the internal cleaning of the paper making structure. It will greatly increase the development cost of recycling equipment. Therefore, the team members unanimously chose option two to continue the subsequent design work.

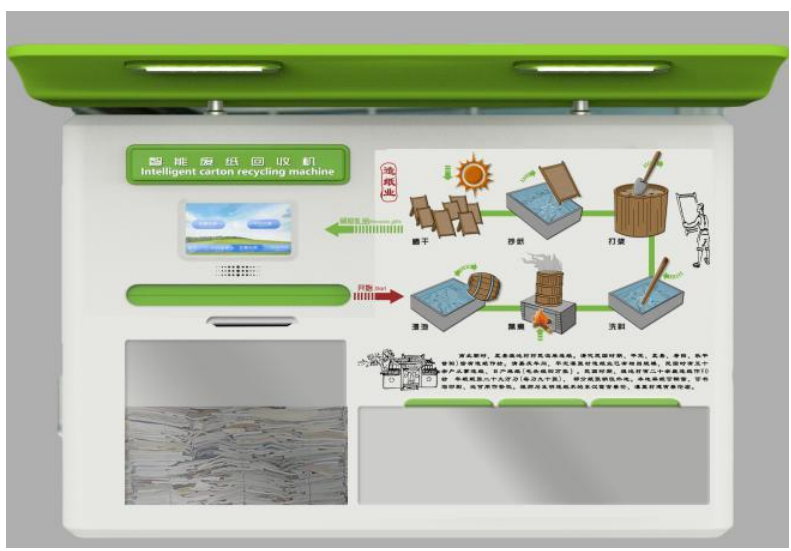

Figure 3: Product plan one

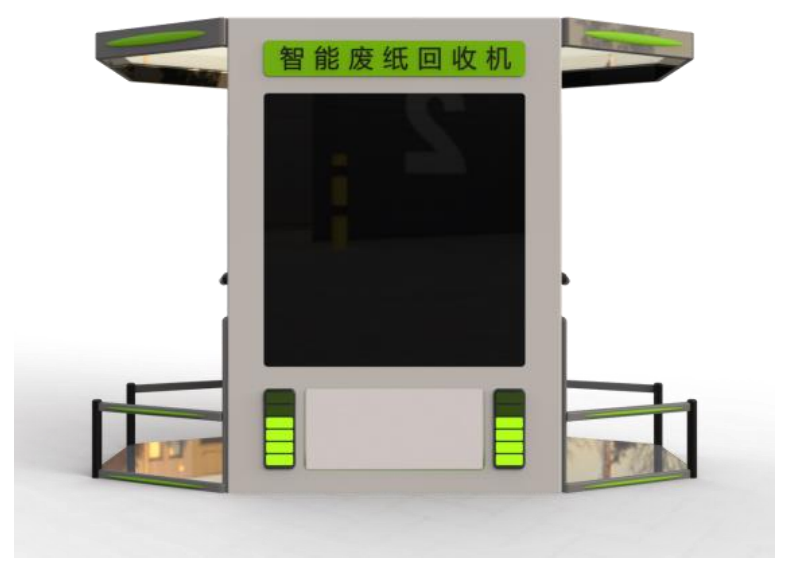

Figure 4: Product plan two

\subsection{The "prototype realization" stage}

"Prototype prototype realization" needs to quickly make a visual product prototype and use the prototype for display, analysis and testing, which can reflect whether the conceptual plan is reasonable and feasible, so that the designer can get feedback as soon as possible and make the final correction and improvement of the product plan. In the "Prototype" step, our team members mainly use 3D geometric models to show the structure, shape and texture of the product. Perform technical analysis on the 3D model such as force analysis and assembly accuracy analysis, and further imitate the use of the product in real life scenarios
(Figure 5/Figure 6).

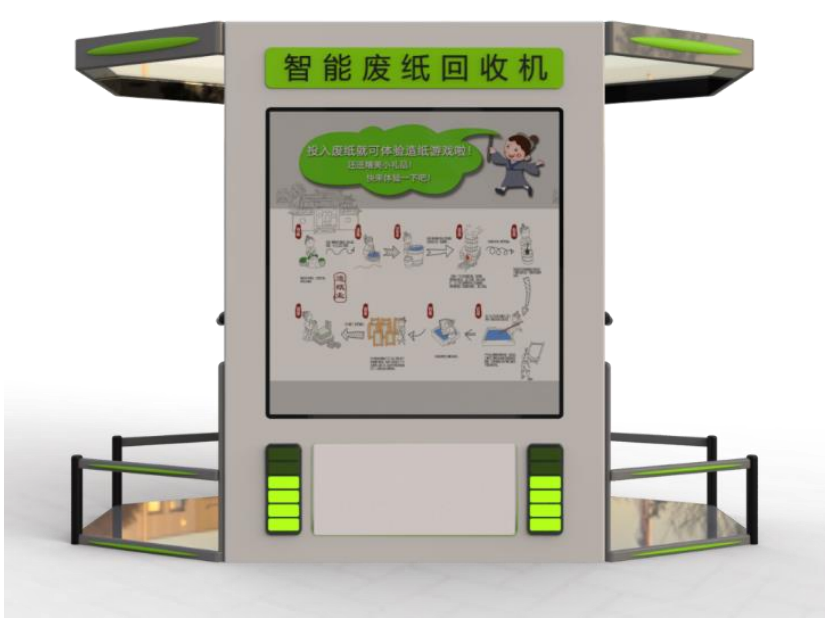

Figure 5: Product overall shape display diagram

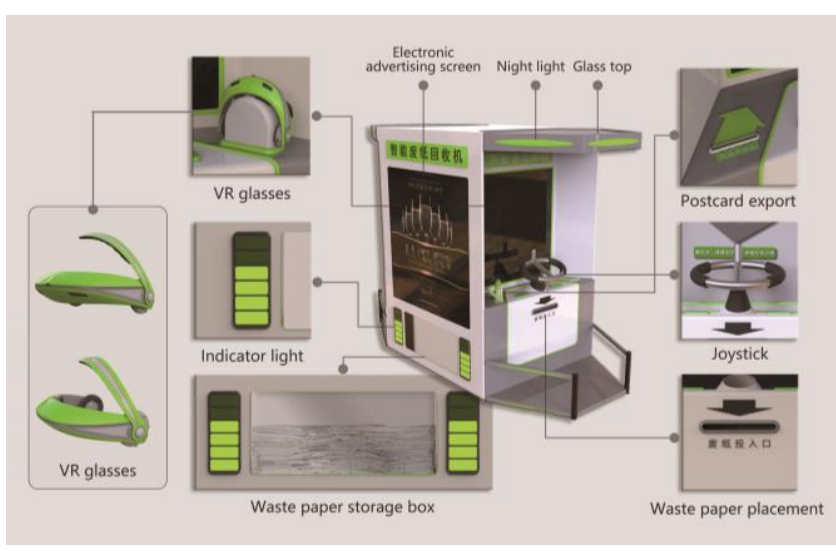

Figure 6: Product structure and details

\subsection{The "Practical Testing" stage}

The "Practical Testing" stage is a stage of perfect design, the target user will test the smart waste paper recycling machine in this stage, and some feedback information is obtained by observing the user's participation experience process and asking open questions. It was difficult to conduct actual user testing since the project had not yet built a physical prototype, so the team adopted the method that invite local citizens, students and shopping mall consumers to "experience" and comment on the project's solution. During the test, the team members sorted out, analyzed and extracted some effective user feedback. Taking into account the user's age and height difference and the user's man-machine comfort when using the product, the design team replaced the fixed hardware interactive interface with a vertically lift able interactive platform (Figure 7). Finally, in order to make the product more beautiful in appearance, more reasonable in structure, and closer to the actual needs of users, we have made overall adjustments to the product size, and further optimized the color matching and product details of the product (Figure 8). In this way, the convenience and interest of users in actual operation can be enhanced. 


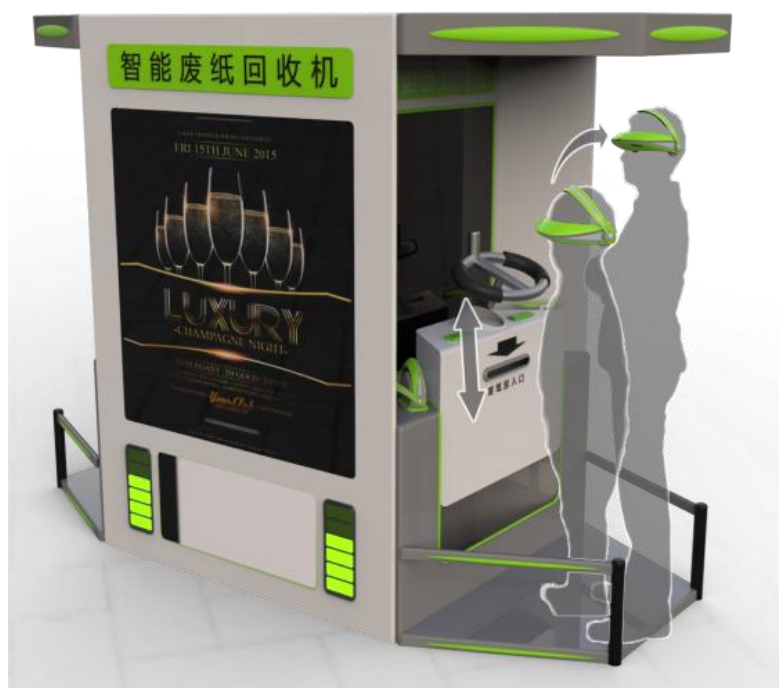

Figure 7: User usage scene graph

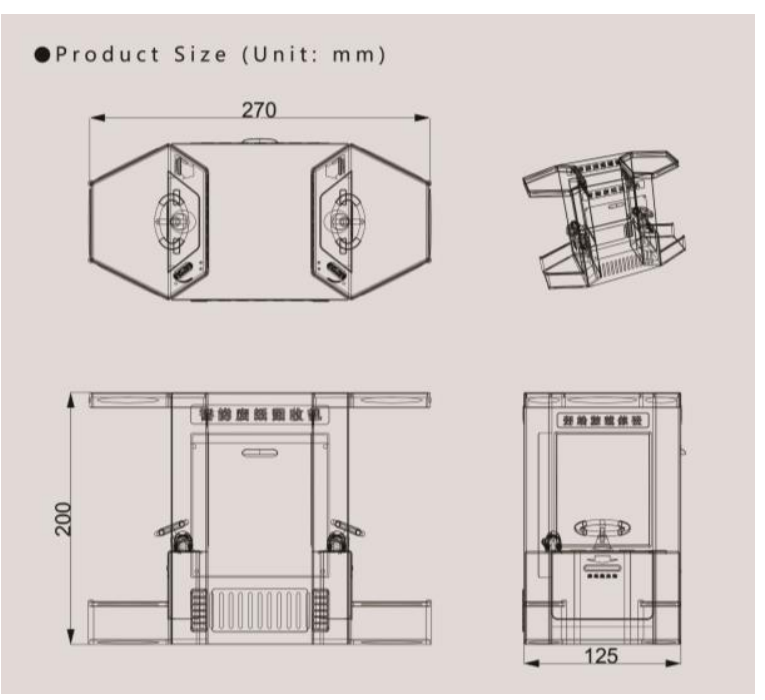

Figure 8: Product size chart

\section{CONCLUSION}

Product innovation design thinking and methods have a very important role and significance for product design. It has different thinking characteristics and requirements from other fields. It can not only improve the creative ability of designers, but also feedback and encourage people to think further to think further to trigger new creative activities. Only when designers master systematic and complete innovative design thinking and methods, and combine specific practices to apply these innovative design methods flexibly, can they consider problems in an all-round and avoid contingency in the design process to further raise novel questions and excellent conceptual scheme.

\section{ACKNOWLEDGMENTS}

First of all, I would like to express my gratitude to all those who helped me during the writing of this thesis. I gratefully acknowledge the help of my supervisor, Mr Han, who has offered me valuable suggestions in the academic studies. In the preparation of this thesis, he has spent much time reading through each draft and provided me with inspiring advice. Second, I also owe a special debt of gratitude to all the professors in Institute Wuhan Institute of Technology, from whose devoted teaching and enlightening lectures I have benefited a lot and academically prepared for the thesis. Last, I should finally like to express my gratitude to my beloved parents who have always been helping me out of difficulties and supporting without a word of complaint.

\section{REFERENCES}

1. Zhang Jiaqi, Fan Junjie. (2009). Application of Innovative Design in Product Design. Art and Design (Theory), 2(10): 234-236.

2. Yang Huifang.(2020). The combination and application of traditional culture and clothing under innovative thinking. Textile Report,(01):117-119.

3. Liang Yuan. (2019). Application research of innovative design methods in product design. Industrial Design,(10):138-139.

4. Yang Zongde. (2001). Innovative thinking methods in the process of innovative design. Mechanical Research and Application, (S1): 52-54. National Machinery Industry Design Innovation Competition 